\title{
Experimental Evaluation of a Wireless Community Mesh Network
}

\author{
Llorenç Cerdà-Alabern, Axel Neumann and Pau Escrich* \\ Universitat Politècnica de Catalunya, Dept. D'Arquitectura de Computadors \\ Jordi Girona 1-3, E-08034 Barcelona, Spain \\ *Guifi.net Foundation, Research group \\ llorenc@ac.upc.edu, neumann@cgws.de, pau.escrich@Guifi.net
}

\begin{abstract}
Nowadays there are inexpensive WiFi devices that have fostered the deployment of wireless communities. Well known routing protocols used in the Internet do not fit well to time varying characteristic of wireless networks. This has motivated an intensive research on routing protocols for wireless mesh networks. At this time there are a number of mature and stable implementations that are being deployed in production networks. In this paper we focus on the experimental evaluation of a production Wireless Mesh network being deployed in a testbed at Universitat Politècnica de Catalunya (UPC) and a quarter of the city of Barcelona, Spain. To our best knowledge, this is the first paper where a production community wireless network using 802.11an is analyzed.
\end{abstract}

\section{INTRODUCTION}

Community networks are deployed and maintained by their own users. Unlike the model used by the traditional telecommunication companies (which are business-focused), each user is owner of a part of the total infrastructure which builds the mesh network. Using an organization system (i.e. web site) they are able to understand each other and connect with neighbors, neighbors of neighbors and so on. These networks are normally open and free. Most of them use WiFi technology because it is the easiest and cheapest way to deploy an outdoor network.

A relevant example is Guifi.net [4, 29, 22, 35], the largest currently existing community network, having around 20.000 operative nodes. Guifi.net has been deployed in urban and rural areas of Spain (mostly Catalonia). Probably one of the main reasons of Guifi.net's success is the fact that it operates as an umbrella for many other small communities. Each community uses its own kind of hardware, software and organization methods (meetings, mailing lists, etc.). But all of them share probably the most important part of the Guifi.net community, the web page. It is used mainly to distribute the IPs and confederate the small networks using a common system.

Guifi.net has become a rather complex and heterogeneous network, merging wired and wireless links. Most of Guifi.net's infrastructure is wireless and the OSPF and BGP routing protocols predominate in the network.

Inside the Quick Mesh Project (QMP) [7] an OpenWRT [5] distribution is being developed using BMX6 as routing protocol [28]. This distribution has been adopted by one of the network communities which operate under the Guifi.net umbrella in the quarter of Sants in Barcelona, Spain. For the sake of brevity we shall refer to the quarter of Sants simply as Sants in the rest of the paper. Additionally, the EU CONFINE project [1] is deploying a research testbed at Universitat Politècnica de Catalunya (UPC) using QMP. CONFINE testbed has been linked to Sants, creating a network referred to as $Q M P S U$ (from Quick Mesh Project at Sants UPC) ${ }^{1}$. The QMPSU network model is expanding among other districts of Barcelona. It grows very fast and other Guifi.net groups in the city have become interested, and started the migration.

In this paper we present an experimental evaluation of QMPSU. To do so, live measurements have been taken hourly over the last 5 months. We use this data to analyze main aspects of QMPSU. These include topology, usage and network performance and characterization. To our best knowledge, this is the first academic work where a production community wireless network using 802.11an is analyzed.

The rest of the paper is organized as follows: Related work is discussed in section 2. Then, some more details about QMP project and QMPSU are given in sections 3 and 4 . The measurements methodology is explained in section 5, followed by the analysis of the topology, Internet access and Usage in sections 6, 7 and 8. Then, wireless links are characterized in section 9 , and some concluding remarks are given in section 10 .

\section{RELATED WORK}

A lot of research has been done in recent years about wireless mesh networks, including design aspects (routing, scalability, security $[28,33,25,38,13,8,27,32,31,34,9]$ ), deployment (urban, rural, centrally-, individually-, or un-planned

\footnotetext{
${ }^{1}$ QMPSU web site: http://Guifisants.net.
} 
$[14,18,23,15])$, measurements and analysis (topologies, performance, usage patterns, evolution, mobility [22, 35, 36, 26, $15,11,10,16,30,12,14,17,18,23])$, as well as surveys of prior work and related aspects [37, 19, 24, 29, 20].

The work presented by Brik [15] about the MadMesh network and Afansasyev [10] about the Google network in Mountain View, California present urban deployments and performance properties of large scale (several hundreds of nodes) mesh networks based on proprietary and centrally managed node systems. In contrast, the system we present is fully open source (GPL licensed) and can be deployed, managed, and maintained completely decentralized. The networks studied by both works are up to 10 times larger than the network studied in this work. While the topology in their networks is organized in a tree-like structure where each mesh node is bound to a parent node upstream to the few GW nodes where all out and incoming traffic aggregates, the topology of our network is managed in a flat (full mesh) structure where each node may become a GW node and/or a relay towards any other node. While the main focus of [10] is about usage patterns, both works present interesting and comparable performance characteristics about the links and end-to-end routes for their backbone networks.

Recent studies of Guifi net include various aspects of network and link characteristics [35, 22, 36], power laws [22], usage patterns, social participation, and evolution over the last 10 years [36]. Like in our work, links are planned individually (decentralized) but the average link length in Guifi.net is (due to its deployment also in rural environments) typically more than 10 times longer than in the QMPSU cloud. Unlike in our system, nodes in Guifi.net are clustered into zones and in fact the network presented in this work is one of these clouds. The studies distinguish between the Guifi core network (or backbone network) which includes only non-leaf nodes and the base network (which including all nodes).

In [26], LaCurts present measurement and analysis of 110 real-world 802.11 mesh networks with an average size of 13 nodes (total of 1407 APs). The focus of this paper is on link-level measurements, investigating SNR versus bit-rate correlation, benefits of opportunistic routing, and the impact of hidden stations. In contrast to our network, only $2.4 \mathrm{GHz} 802.11 \mathrm{bgn}$ standard has been used (QMPUS also uses $5 \mathrm{GHz} 11 \mathrm{an}$ ) for mostly indoor deployments and few measurements are given about end-to-end performance in the studied networks.

Pioneering work on the Roofnet testbed by Bicket and Aguayo $[12,14]$ and on the TFA-Mesh in Houston TX by Camp $[18,17]$ are analyzing the multi-hop performance of purely $802.11 \mathrm{~b}$ based mesh networks. While the size of these networks are in the same order as the QMPSU network (37 and 18 nodes respectively and up to 69 nodes in QMPSU), the density of Roofnet with 344 edges and a median of 18 links per node is outstanding and a consequence of the unplanned deployment with only omni-directional antennas.

\section{QUICK MESH PROJECT}

QMP [7] is an operating system for embedded network devices based on OpenWRT/Linux. It was started by some Guifi.net activists in 2011 with the objective to provide a fully open-source solution to easily and quickly deploy a mesh network and share Internet uplinks between it's users.

As main routing protocol QMP uses BMX6 [28], a destination-sequenced distance-vector protocol using UDP messages to discover other nodes and disseminate node and routing information. Some extra features have been specially developed for QMP, such as a smart gateway selection using IPIP tunnels or a short message plugin which permits to send arbitrary information to other nodes, piggybacked by the protocol packages. BMX6 obtained the best results over other mesh routing protocols tested in the Wireless Battle Mesh v6 celebrated in Aalborg (Denmark). Other important characteristics of QMP are the native IPv6 and full auto-configuration support. Each node auto-configures its own IPv6 address based on a ULA ${ }^{2}$ prefix. IPv4 connections are enabled via tunnels over the ULA-based IPv6 network.

\section{QMPSU ARCHITECTURE}

QMPSU is a wireless, 802.11-based, mulit-hop, semi-static, and productively used mesh network where (topologically) all nodes provide the same routing functionality for relaying other node's traffic. Most of the network users only have the mesh network for reaching the internet, so they depend on the community. In consequence, stability and good performance of the network are mandatory points. QMPSU is not a pure MANET network, it is being deployed following a model named by the participants: SmartMesh. The concept tries to explain a model placed between the complete unmanaged model used in many of the known Mesh/MANET networks and the complete organized model used in the AP/Infrastructure networks. The main points which define the SmartMesh deployment idea are:

- Use patch antennas with $<90^{\circ}$ as main equipment in AD-HOC mode.

- Use parabolic antennas $\left(<6^{\circ}\right)$ for long shots (pointto-point links).

- Use two or more devices for some strategic locations (super-nodes).

- Use different channels to multiplex the spectrum (super-nodes make the interconnection).

- Use only 802.11an to reduce the noise and interferences (spectrum space is bigger than 802.11bgn).

- Use only stable links and devices placed in the outdoor (roof or balcony).

These requirements imply the inconvenience of planning in advance the deployments and links and requires to manually orientate antennas during the installation. But it has the advantage of reduced interference (one of the biggest problems in ISM-spectrum based networks) and fewer neighboring nodes but with better links. Following this model, reasonable end-to-end performance could be achieved even over 7 ore more hops.

\footnotetext{
${ }^{2}$ RFC4193 Unique Local Address
} 


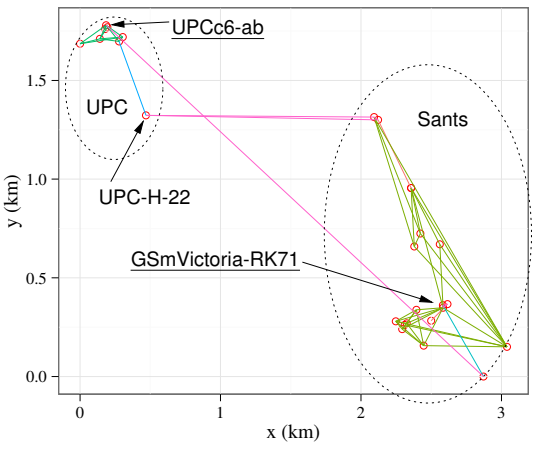

Figure 1: QMPSU network. main gateways are underlined.

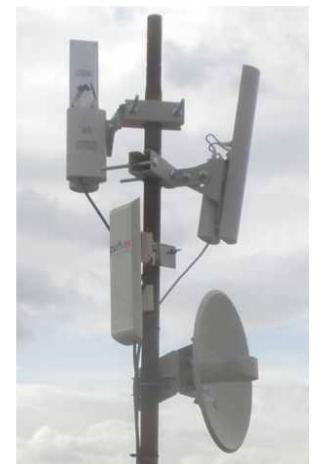

Two Figure 2: Supernode.

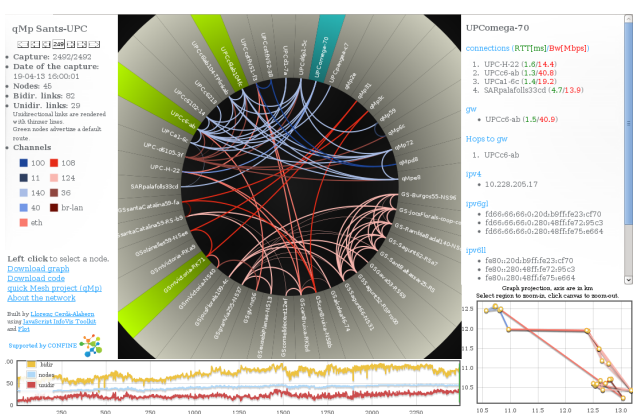

Figure 3: QMPSU network web page.
The most common hardware used in QMPSU is NanoStation $\mathrm{M}^{3}$, which has the following characteristics: Antenna $5 \mathrm{GHz}$ $16 \mathrm{dBi}$, Processor Atheros MIPS $400 \mathrm{MHz}$, Flash Memory 8MB, SDRAM Memory 32MB, two ethernet ports 10/100, Radio Atheros 9k 802.11AN MiMo 2T2R. For point-to-point long shots the typically used hardware is NanoBridge $\mathrm{M} 5^{4}$, a variant of the NanoStation with parabolic antenna. Finally for super-nodes, the most common equipment is a couple of Rocket $M 5^{5}$ with $120^{\circ}$ sector antennas (see figure 2).

\section{METHODOLOGY}

Measurements were obtained using ssh to connect to each QMPSU node and gathering information with basic system commands available in the QMP distribution. This method has the advantage that no changes or additional software had to be installed in the nodes. This is an important point, since being a community network, the users are the owner of their nodes, and so, a minimum intrusion was desirable. The data collection was done hourly from December the 29th 2012 to June the 13th 2013. A simple monitoring web page was developed, which is publicly available at [6] (see figure 3). The web page allows navigating through the graphs obtained in the captures.

QMPSU is rather dynamic. Several reasons contribute to this fact: Being a community network the growth is essentially unplanned. In Sants, nodes are added by community members using their home roofs, which are often at non optimal locations. This fact produce a high diversity on the quality of the links, making some of them to flip-flop time to time, and even some nodes to be sporadically unreachable. Other reasons of unreachability have been electricity cuts, nodes that have been upgraded, reconfigured, hanged, etc. Additionally, community members some times have re-tuned the radios of their devices, trying to achieve better performance (transmission power, channel and other parameters), thus, changing the characteristics of the links. Furthermore, during the measuring period not only new nodes have been added, but other were removed or changed their position. E.g. testing nodes only used temporarily at UPC, or users

\footnotetext{
3 http://www . ubnt.com/downloads/datasheets/nanostationm/ nsm_ds_web.pdf

${ }^{4}$ http://www . ubnt.com/downloads/datasheets/nanobridgem/ nbm_ds_web.pdf

${ }^{5}$ http://www.ubnt.com/downloads/datasheets/rocketmgps/ Rocket_M_GPS_Datasheet.pdf
}

that changed residence in Sants.

Characterizing such a dynamic network is challenging. That is why in the following sections we have chosen different statistical representations depending on the parameter under study. For instance, in order to reflect the dispersion of the measured parameters, in some cases we have used standard boxplots (showing the range, median and quartiles).

\section{QMPSU TOPOLOGY}

Figure 4 shows the number of nodes and bidirectional and unidirectional links found in each capture. Regarding the links, we have considered those reported by BMX6 (with the command bmx6 -c show=links). For bidirectional links, in figure 4 we count both links in opposite direction as a single link. Figure 5 shows the nodes and links presence. We define presence as the percentage of times a given node or link is observed over the captures.

Figure 4 shows that QMPSU is growing. The solid line in the figure shows a linear regression fit, which increases from around 33 to 48 nodes. On the average 40.6 nodes have been found, 21.4 in Sants and 19.2 in UPC. Even if UPC and Sants have a similar number of nodes, at UPC the nodes are distributed in the Campus, which covers a rather smaller area than Sants (see figure 1). Overall, 69 different nodes where detected. From those, figure 5 shows that only 42 had a presence larger than $50 \%$. Indeed, figure 5 shows that only 27 nodes were alive almost all measuring period, having a presence higher than $80 \%$.

Figure 6 shows the link length Complementary Empirical Cumulative Distribution Function (CECDF). We have found that the link length distribution can be fitted by a mixture of 2 exponentials (solid line in figure 6). The distribution is fitted with correlation coefficient of $\rho=0.996$. This is in line with the results reported for Guifi.net in [21, 35]. Let $L$ be the complementary CDF of the link length, $X$, then: $L\left(x \mid \mu_{1}, \mu_{2}, \theta\right)=P(X>x)=\theta \mathrm{e}^{-x / \mu_{1}}+(1-\theta) \mathrm{e}^{-x / \mu_{2}}$ This result shows that links can be grouped in two sets: $42 \%$ of shorter links with mean $\mu_{1}=196 \mathrm{~m}$ and $58 \%$ of longer distance links with mean $\mu_{2}=581 \mathrm{~m}$. Thus, an overall mean link length of $419.3 \mathrm{~m}$.

Figure 7 shows the out degree distribution. To derive this figure we have proceeded as follows: We have first built 


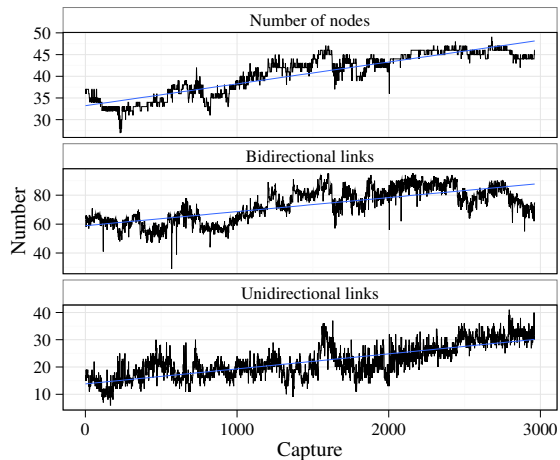

Figure 4: Number of nodes, bidirectional and unidirectional links in each capture.

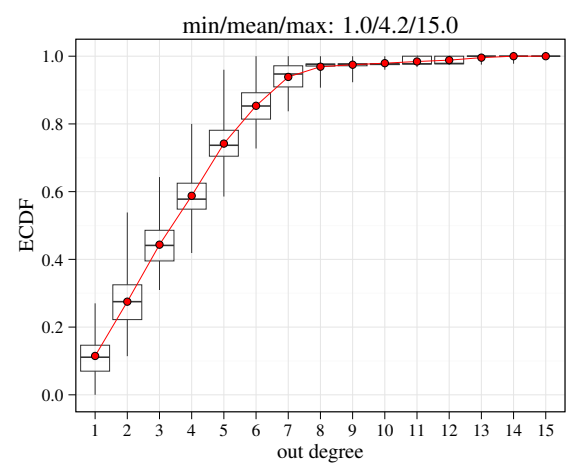

Figure 7: Average out degree ECDF.

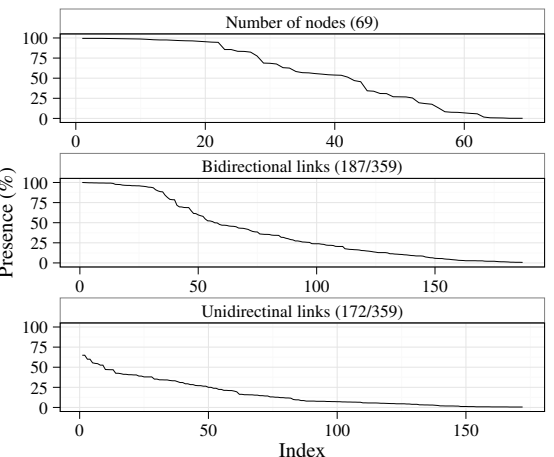

Figure 5: Nodes and links presence.

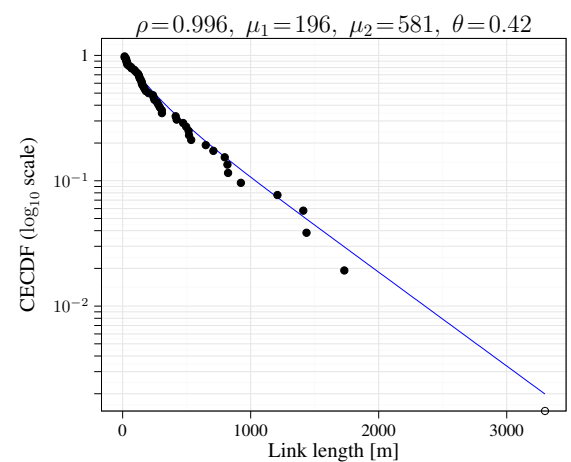

Figure 6: Link length distribution.

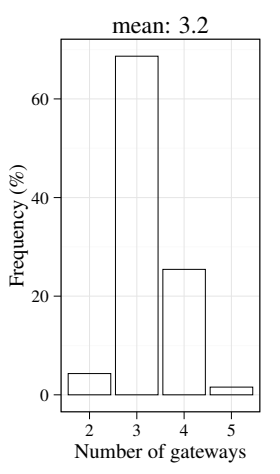

Figure 8: Number of gateways.

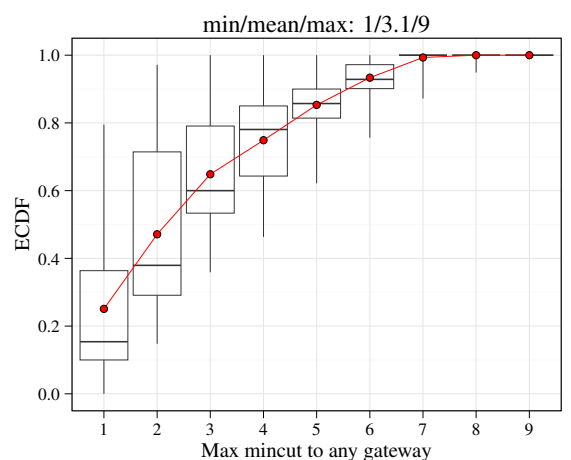

Figure 9: Average ECDF of the max-mincut from a node to any of its gateways. the graph of each capture and its out degree ECDF. Note that the graphs may change in every capture for the reasons formerly described. Then, for each out degree we have taken the average of its ECDF value obtained over all captures. In order to show the variability of the out degree, in figure 7 we have added a boxplot of the out degree ECDF values obtained over the captures.

Figure 7 shows that, on the average, around $90 \%$ of the nodes have more than 1 link, and around $40 \%$ of the nodes have at least 4 links, with an overall average degree of 4.2 . This is in contrast with Guifinet[35], were the average is around 2, and only $20 \%$ of the nodes have degree higher than 3. This fact can be explained by the higher number of links that are automatically discovered and established by the nodes in the ad-hoc configuration used in QMPSU, than the static links manually configured in Guifi.net. We note that no standard distribution (including a power law) has been found to fit the average out degree. Therefore, it cannot be stated that the scale-free pattern found in the Internet applies to QMPSU. Nevertheless, the size of QMPSU (around 45 nodes) is too small to do an accurate topology fitting.

\section{INTERNET ACCESS}

Internet access is provided by some nodes of QMPSU having links with other nodes of Guifi.net. These nodes disseminate a default route, and we shall refer to them as gateways. The number of gateways has been variable during the measur- ing period: there were found between 2 and 5 gateways, 3.3 on the average. Figure 8 shows the frequency plot of the number of gateways. BMX6 estimates a metric to each gateway, and chooses the best one. Note that when a gateway becomes unreachable by a node, it will stop receiving its default route announcements, and BMX6 will switch to another one. Thus, in order for a node to be disconnected from the Internet, is necessary a failure in all links that allows the node to reach any of the gateways. Therefore, as an estimation of robustness of the Internet availability we define the max-min-cut to any gateway as the maximum min-cut between a node and any gateway of the network. Recall that the min-cut between two nodes $s$ and $t$ is the minimum number of edges such that, after removal, there is not directed path from $s$ to $t .^{6}$ Figure 9 shows the average ECDF of the max-min-cuts obtained in each capture. The distribution has been obtained following the same procedure as in figure 7 , i.e. taking the average of the max-min-cut ECDF obtained for each graph, and adding a boxplot in order to show the dispersion of each value. Figure 9 shows that around $75 \%$ of the nodes have a max-min-cut of at least 2 links to a gateway. We note that no standard distribution have been found to fit the average max-min-cut. Figure 8 shows that the dispersion of the max-min-cut equal to 2 is rather high: the interquartile of the ECDF values for this point is around 0.42 . This is motivated by the fact that one of the nodes at UPC (called UPC-H-22, see figure 1) had a

\footnotetext{
${ }^{6}$ There are efficient algorithms to compute the s-t min-cut available in common numerical tools.
} 


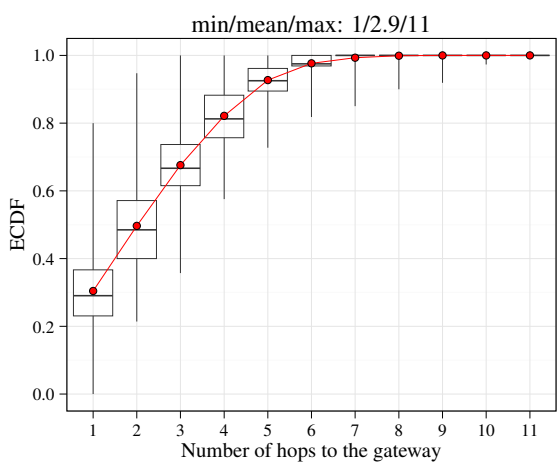

Figure 10: Average ECDF of the number of hops to the gateway.

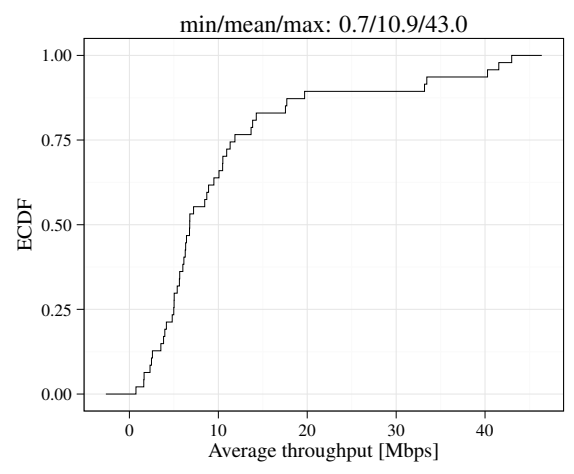

Figure 11: ECDF of the average throughput to the gateway.

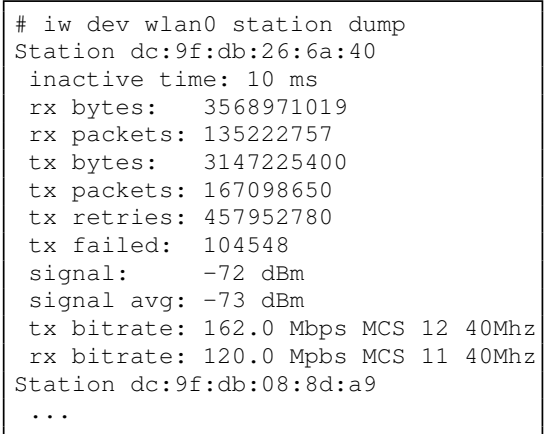

Figure 13: Output of the command iw dump.

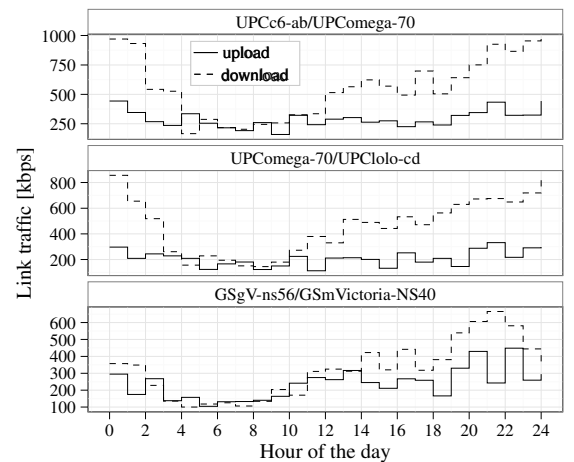

Figure 16: Traffic in the 3 busiest links.

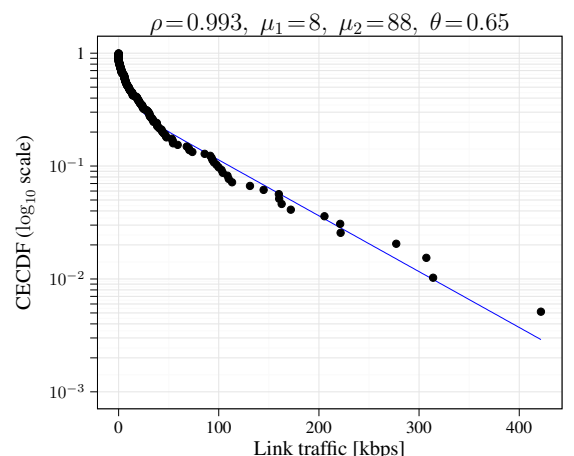

Figure 14: Link traffic CECDF.

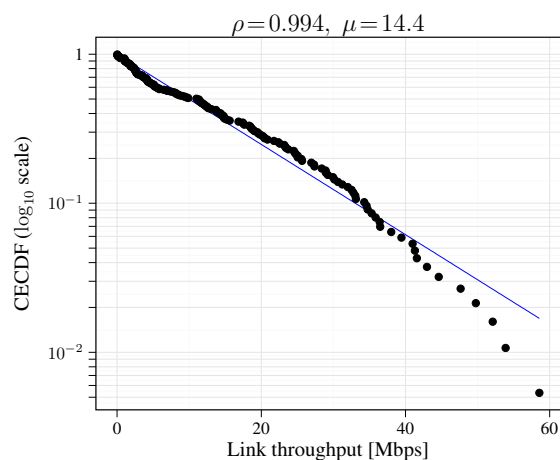

Figure 17: Throughput CECDF.

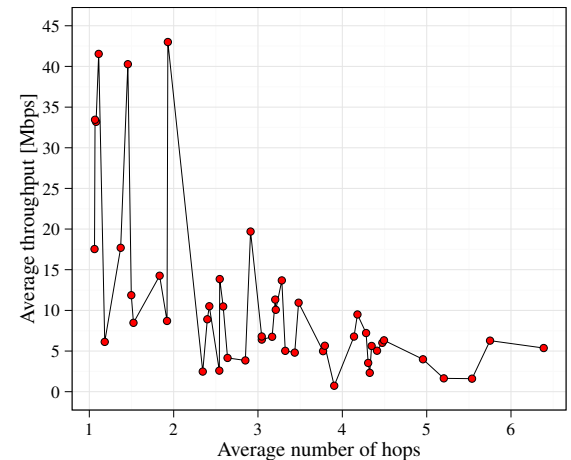

Figure 12: Average throughput vs. average number of hops to the gateway.

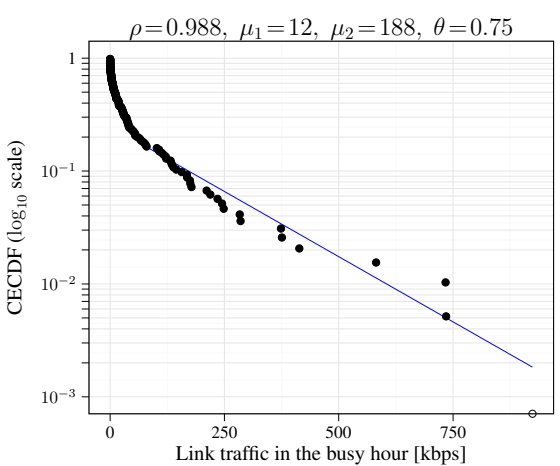

Figure 15: Link traffic in the busy hour CECDF.

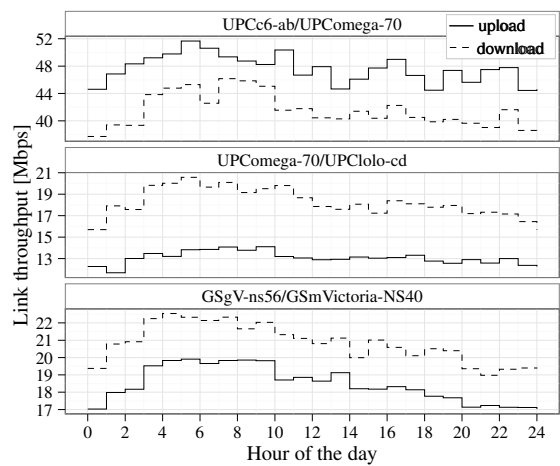

Figure 18: Throughput in the 3 busiest links. wireless link not very stable with the Sants. The failure of this link decreases the max-min-cut of many nodes.

Figure 10 depicts the average ECDF of the number of hops to the gateway of each node. This has been derived from the routing tables, which were also recorded in every capture. The plot has been obtained similarly to figures 7 and 9, i.e. taking the average of the ECDF obtained for each capture, and adding a boxplot in order to show the dispersion. Figure 10 shows that around $67 \%$ of the nodes have 3 or less hops to the gateway, with an average of 2.9 hops. We can see from the boxplots of the figure that the ECDF measured over the captures does not show strong deviations.
We have estimated links throughput using the TCP_STREAM test of netperf [3]. As before, measurements were taken hourly. The command was run from every node to its gateway. In order to limit the disturbance to the users we tried to reduce the test to the minimum time. After some trials, we observed that running netperf tests of only 3 seconds yield a good estimation. The throughput of every wireless link was also computed (link throughputs are discussed in section 9). For the link measurements IPv6 link local addresses were used, thus, assuring that no other links would be used. To avoid interferences, throughputs tests were done in serial (only one test at a time). Figure 11 depicts the ECDF of the 

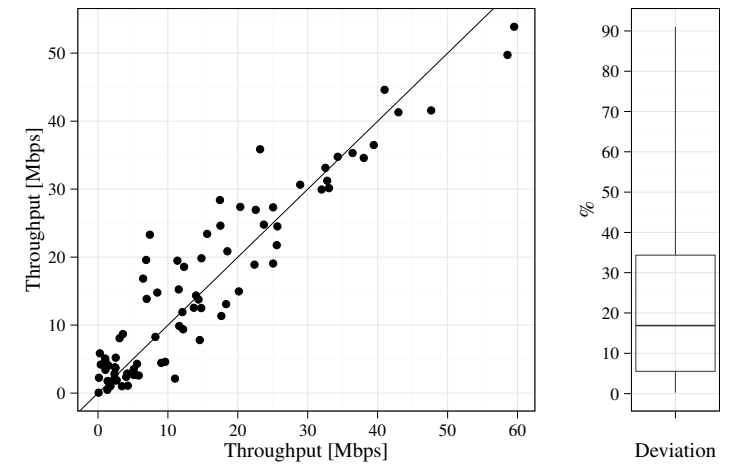

Figure 19: Throughput asymmetry.
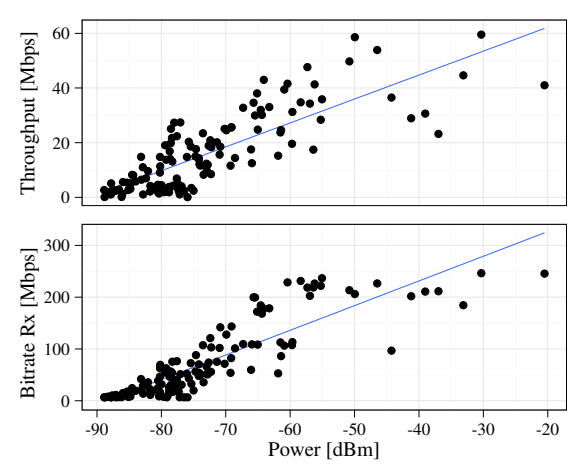

Figure 20: Throughput (top) and average bitrate of received packets (bottom) vs signal power.

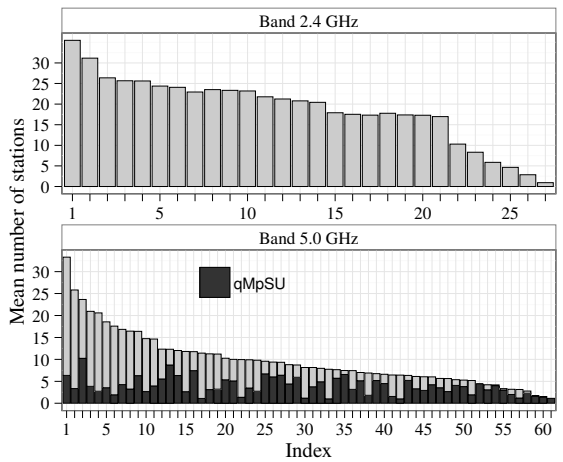

Figure 21: Number of stations per scan.

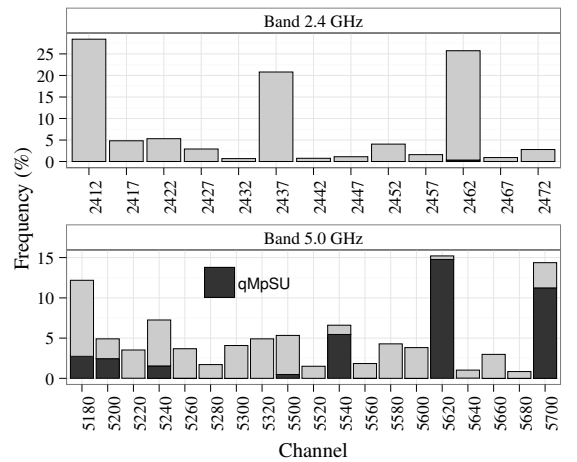

Figure 22: Channel occupancy.

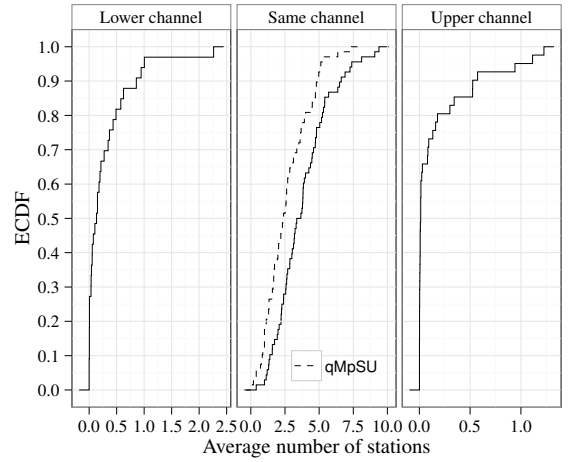

Figure 23: ECDF of the number of interfering stations. average throughput of the nodes to their gateway measured over all captures. The figure shows that the throughputs are rather high, with an average of $10.9 \mathrm{Mbps}$. This is due to the high performance that can be achieved with MIMO 802.11an cards of most equipment. Finally, figure 12 shows the average throughput vs. average number of hops to the gateway. As expected, throughput tend to reduce as the number of hops increases. However, the line is rather irregular, due to the diversity of the links.

\section{QMPSU USAGE}

We have gathered the usage of the network using the linux iw command [2] (see figure 13). Recall that captures were done hourly. Thus, taking the difference between the transmitted bytes counter ( $\mathrm{tx}$ bytes in figure 13) of two consecutive runs of $i w$, it is possible to estimate the average traffic sent each hour in every link. Measurements where done using directional links, i.e. traffic sent in opposite directions between the same nodes is counted as two different links. Only wireless links, and having a presence higher than $25 \%$ (in order to avoid outliers) where considered in the statistics: 196 links in total. Figure 14 shows the CECDF of the average traffic sent in each of these links. Interestingly, it was found that the traffic is well fitted by a mixture of 2 exponentials (solid line in figure 14): $65 \%$ with mean $\mu_{1}=8 \mathrm{kbps}$ and $35 \%$ with mean $\mu_{2}=88 \mathrm{kbps}$ (overall mean of $36 \mathrm{kbps}$ ). An explanation of this result is the presence of two groups of links: Those where most of the traffic belongs to a single user, and backbone links carrying the aggregate traffic from a number of users. We found that the hour having the highest average traffic (busy hour) was between $22 \mathrm{~h}$ and $23 \mathrm{~h}$. Figure 15 shows the CECDF of the average traffic sent in the busy hour. The figure shows that a mixture of 2 exponentials still gives a good fit. The overall average is now $56 \mathrm{kbps}$, almost $55 \%$ higher than before. Figure 16 shows the average traffic in both directions of the three busiest links over each hour of the day.

\section{QMPSU WIRELESS LINKS}

In this section we try to characterize the wireless links of the network. We start by studying their throughput, measured using netperf as described in section 7 . In order to avoid outliers, only those of section 8 are considered. From the 196 links, the throughput was able to be measured in $169(86 \%)$. Failures were due to unidirectional links (were netperf cannot run the test), or too weak links for netperf to succeed. Figure 17 shows the CECDF of the throughput of the links were netperf succeeded. The figure shows that the link throughput can be fitted with an exponential distribution with mean 14.4 Mbps. In order to see the variability of the throughput, figure 18 shows the throughput averages in both directions of the three busiest links (same links as in figure 16) over each hour of the day. Comparing figures 18 and 16 we observe that the throughput is only slightly affected by the traffic in the links. Additionally, measurements on each direction of the links are identified with the same solid or dashed lines in figures 15 and 16. Comparing the figures it is apparent that the asymmetry of the throughputs measured in both directions it is not due to the asymmetry of the users' traffic. For instance, around 5am, when the 
user traffic is the lowest and equal in both directions, the asymmetry of the links throughputs observed in figure 15 remains the same. We thus conclude that this asymmetry must be links characteristics, as level of interferences present at each end, or different transmission powers.

In order to measure the links' asymmetry, figure 19 depicts the throughput measured in each direction. A boxplot of the absolute value of the deviation over the mean is also depicted on the right. The figure shows that around $25 \%$ of the links have a deviation higher than $30 \%$. Thus, we can conclude that the symmetry of the links, an assumption often used in the literature of in wireless mesh networks, is not very realistic.

Figure 20 shows the average throughput of each link (top of the figure) versus the average signal power of the received packets (measured with iw dump). The figure also show the average bitrate reported by iw dump for unicast received packets (bottom of the figure). We have assumed that unicast transmissions correspond to packets with bitrates higher than the lowest basic rate $(6 \mathrm{Mbps}$ in the $5 \mathrm{GHz}$ band). As expected, the figure shows the clear dependency of both measures with respect of the signal power.

Finally, we have estimated the interference level by doing a scan in every node at each hourly capture. All nodes were configured in ad-hoc mode in the $5 \mathrm{GHz}$ band. However, some radios were dual band, and reported scans in both 2.4 and $5 \mathrm{GHz}$. We discriminated QMPSU stations because they share the same BSSID address, which is reported in the scans. Figure 21 shows the average number of stations detected in every scan of the nodes, in decreasing order. The figure shows the stations detected in both bands. The average of QMPSU stations is shown in darker color. Figure 21 shows that the $2.4 \mathrm{GHz}$ band is much more crowded: only 3 non-overlapping channels of $20 \mathrm{MHz}$ are available, while a much higher number of stations are detected. Figure 23 shows the number of stations detected on every channel. As expected, it can be observed as most of the stations detected in the $2.4 \mathrm{GHz}$ band use the recommended 3 non-overlapping channels. In the $5 \mathrm{GHz}$ it can be observed the channel where most of the QMPSU stations are located. Note that it is not possible to have high frequency diversity in QMPSU since most of the stations have only a single radio. Even if channel assignment is a key issue, it was fixed manually by QMPSU users based on trial and error. Figure 23 shows the ECDF of the average number of stations detected in the same and adjacent channels used by QMPSU nodes (5 GHz band). ECDF of detected QMPSU nodes is also reported in dashed lines. All QMPSU nodes were configured to use channel bonding (i.e. combining two adjacent $20 \mathrm{MHz}$ channels). Thus, they can potentially interfere with stations located in adjacent channels. Figure 23 confirm the low number of stations belonging to external networks interfering with QMPSU nodes: around $90 \%$ of nodes detect less than 1 station, on the average, from external networks in each of the 3 channels of interest. Looking at the stations in the same channel, around $50 \%$ detect more than 3.5, from which more than 2.5 are QMPSU.

\section{CONCLUSIONS}

In this work we present an experimental evaluation of QMPSU, a wireless community network deployed at Universitat Politècnica de Catalunya (UPC) and Sants, a quarter of Barcelona, Spain. QMPSU is rather dynamic due to many reasons, e.g.: its community nature in an urban area; it is a growing network; there is a high diversity of the quality of wireless links; the mesh nature of the network.

Characterizing such a dynamic network is challenging. To do so we have performed an extensive statistical study of the main parameters. These include topological properties, Internet access, usage of the network and characterization of the radio links.

We have found simple distributions that fit some of these parameters. For instance, the network is not scale-free, the link length and traffic is fitted by a mixture of two exponentials, and the average throughput of the links is exponentially distributed. Regarding radio links, we have observed a non negligible asymmetry.

Our results show that the network is rather well connected and adaptive. Thus, demonstrating the advantages of a wireless mesh network. Furthermore, even if the network is deployed in an urban area with an average link length of around $500 \mathrm{~m}$, an average link throughput of around $14 \mathrm{Mbps}$ was obtained. This high performance can be attributed to the 802.11an devices used in the network.

\section{Acknowledgments}

This work was supported by the European project CONFINE http://confine-project.eu, and Spanish grants TIN2010-21378-C02-01 and 2009-SGR-1167.

\section{REFERENCES}

[1] Community Networks Testbed for the Future Internet, CONFINE. http://confine-project.eu/. FP7 European Project 288535.

[2] Linux utility for wireless devices. http: //wireless.kernel.org/en/users/Documentation/iw.

[3] Netperf benchmark. http://www.netperf.org/netperf.

[4] Open, Free and Neutral Network Internet for everybody. http://guifi.net/en.

[5] OpenWrt Linux distribution for embedded devices. https://openwrt.org.

[6] qMp Sants-UPC monitoring web page. http: //compnet.ac.upc. edu/ 1 lorenc/qmpsantsupc/index.php.

[7] Quick Mesh Project. http://qmp.cat.

[8] M. Abolhasan, B. Hagelstein, and J.C.-P Wang. Real-world performance of current proactive multi-hop mesh protocols. In 15th Asia-Pacific Conference on Communications (APCC), October 2009.

[9] Loay Abusalah, Ashfaq Khokhar, and Mohsen Guizani. A survey of secure mobile ad hoc routing protocols. In IEEE Communications Surveys and Tutorials, 2008.

[10] M. Afanasyev, T. Chen, G.M. Voelker, and A.C. Snoeren. Usage patterns in an urban wifi network. IEEE/ACM Transactions on Networking, 18(5), 1359-1372, 2010.

[11] Mikhail Afanasyev, Tsuwei Chen, Geoffrey M. Voelker, and Alex C. Snoeren. Analysis of a mixed-use 
urban wifi network: When metropolitan becomes neapolitan. In In ACM/USENIX IMC, 2008.

[12] D. Aguayo, J. Bicket, S. Biswas, G. Judd, and R. Morris. Link-level measurements from an $802.11 \mathrm{~b}$ mesh network. ACM SIGCOMM Computer Communication Review, 34(4), 121-132, 2004.

[13] U. Ashraf, G. Juanole, and S. Abdellatif. Evaluating routing protocols for the wireless mesh backbone. In Wireless and Mobile Computing, Networking and Communications (WIMOB), October 2007.

[14] John Bicket, Daniel Aguayo, Sanjit Biswas, and Robert Morris. Architecture and evaluation of an unplanned $802.11 \mathrm{~b}$ mesh network. In Proceedings of the 11th annual international conference on Mobile computing and networking, MobiCom '05, pages 31-42, Cologne, Germany, 2005.

[15] Vladimir Brik, Shravan Rayanchu, Sharad Saha, Sayandeep Sen, Vivek Shrivastava, and Suman Banerjee. A measurement study of a commercial-grade urban wifi mesh. In Proceedings of the 8th ACM SIGCOMM conference on Internet measurement, pages 111-124, 2008.

[16] Vladimir Bychkovsky, Bret Hull, Allen Miu, Hari Balakrishnan, and Samuel Madden. A measurement study of vehicular internet access using in situ wi-fi networks. In Proceedings of the 12th annual international conference on Mobile computing and networking, pages 50-61. ACM, 2006.

[17] Joseph Camp, Vincenzo Mancuso, Omer Gurewitz, and Edward W. Knightly. A measurement study of multiplicative overhead effects in wireless networks. In in Proceedings of IEEE INFOCOM, Uppsala, Sweden, April 2008.

[18] Joseph Camp, Joshua Robinson, Christopher Steger, and Edward Knightly. Measurement driven deployment of a two-tier urban mesh access network. In Proceedings of the 4 th international conference on Mobile systems, applications and services, pages 96-109. ACM, 2006.

[19] G. Camponovo and D. Cerutti. Wlan communities and internet access sharing: a regulatory overview. In Mobile Business, 2005. ICMB 2005. International Conference on, pages 281-287. IEEE, 2005.

[20] Y. Cao, M. Krebs, G. Toubekis, and S. Makram. Mobile community information systems on wireless mesh networks-an opportunity for developing countries and rural areas. In Fifth International Workshop on Ubiquitous Mobile Information and Collaboration Systems (UMICS'07), pages 11-12. Citeseer, 2007.

[21] Llorenç Cerdà-Alabern. Analysis of guifi.net's topology, extension of results. Technical Report UPC-DAC-RR-2012-15, May 2012.

http://www.ac.upc.edu/app/research-reports/html/ 2012/15/guifinet-topology-extension.pdf.

[22] Llorenç Cerdà-Alabern. On the topology characterization of guifi.net. In 2012 IEEE 8th International Conference on Wireless and Mobile Computing, Networking and Communications (WiMob'2012), pages 389-396, Barcelona, Spain, October 2012.

[23] Kameswari Chebrolu, Bhaskaran Raman, and Sayandeep Sen. Long-distance 802.11b links: Performance measurements and experience. In in Proceedings of ACM
Mobicom, 2006.

[24] P.A. Frangoudis, G.C. Polyzos, and V.P. Kemerlis. Wireless community networks: an alternative approach for nomadic broadband network access. Communications Magazine, IEEE, 49(5), 206-213, 2011.

[25] D. Johnson, N. Ntlatlapa, and C. Aichele. Simple pragmatic approach to mesh routing using batman. In $2 n d$ IFIP International Symposium on Wireless Communications and Information Technology in Devel oping Countries, CSIR, Pretoria, South Africa, October 2008.

[26] Katrina LaCurts and Hari Balakrishnan. Measurement and analysis of real-world 802.11 mesh networks. In Proceedings of the 10th Annual Conference on Internet Measurement (ACM), pages 123-136, Melbourne, Australia, 2010.

[27] D. Murray, M.W. Dixon, and T. Koziniec. An experimental comparison of routing protocols in multi hop ad hoc networks. In Australasian Telecommunication Networks and Applications Conference (ATNAC), Auckland, New Zealand, 2010.

[28] Axel Neumann, Ester López, and Leandro Navarro. An evaluation of bmx6 for community wireless networks. In $1 s t$ International Workshop on Community Networks and Bottom-up-Broadband (CNBuB'2012), pages 651-658, Barcelona, Spain, October 2012.

[29] M. Oliver, J. Zuidweg, and M. Batikas. Wireless commons against the digital divide. In Technology and Society (ISTAS), IEEE International Symposium on, pages 457-465. IEEE, 2010.

[30] Joshua Robinson, Ram Swaminathan, and Edward W. Knightly. Assessment of urban-scale wireless networks with a small number of measurements. In The 14th ACM international conference on Mobile computing and networking, MobiCom '08, pages 187-198. ACM, 2008.

[31] Shih-Hao Shen, Yueh-Min Huang, and Jen-Wen Ding. A cross-layer design for heterogeneous routing in wireless mesh networks. In Int. J. Pervasive Computing and Communications, 2008.

[32] Muhammad S. Siddiqui and Choong Seon Hong. Security issues in wireless mesh networks. In IEEE International Conference on Multimedia and Ubiquitous Engineering (MUE'07), 2007.

[33] C. Szabó, Z. Horváth, and K. Farkas. Wireless community networks: motivations, design and business models. In 3rd international conference on Wireless internet, page 23. ICST (Institute for Computer Sciences, Social-Informatics and Telecommunications Engineering), 2007.

[34] Parul Tomar, P.K. Suri, and M. K. Soni. A comparative study for secure routing in manet. In International Journal of Computer Applications, 2010.

[35] Davide Vega, Llorenç Cerdà-Alabern, Leandro Navarro, and Roc Meseguer. Topology patterns of a community network: Guifi.net. In 1st International Workshop on Community Networks and Bottom-up-Broadband (CNBuB'2012), pages 612-619, Barcelona, Spain, October 2012.

[36] Davide Vega, Roc Meseguer, Leandro Navarro, and Felix Freitag. Measurement and analysis of a large-scale wireless community network. In Submitted for SIGCOMM 2013 Barcelona, Barcelona, Spain, 2013.

[37] S. Vural, Dali Wei, and K. Moessner. Survey of experimental evaluation studies for wireless mesh network deployments in urban areas towards ubiquitous internet. Communications Surveys Tutorials, IEEE, 15(1), 223-239, 2013.

[38] A. Zakrzewska, L. Koszalka, and I. Pozniak-Koszalka. Performance study of routing protocols for wireless mesh networks. In Systems Engineering, 2008. ICSENG '08. 19th International Conference, August 2008. 